\title{
NIVEL DE INTERACCIÓN DE LA COMUNICACIÓN DE LOS PARTIDOS POLÍTICOS ESPAÑOLES EN REDES SOCIALES
}

\author{
Level of Iteraction of the Communication of Spanish Political Parties in Social Networks
}

\author{
M NEREIDA CEA ESTERUELAS \\ Universidad de Málaga \\ marceaest@uma.es
}

Resumen: Las redes sociales juegan un importante papel en la difusión de mensajes, no solo durante periodos electorales, sino también en toda estrategia de comunicación política. El objetivo de este trabajo es analizar la comunicación de los principales partidos políticos españoles en las redes sociales desde el punto de vista de los estudios de recepción. Para ello se analiza la respuesta de la audiencia a los mensajes lanzados por partidos políticos durante un mes, en las cuatro principales redes sociales (Twitter, Facebook, Youtube e Instagram). Para tal fin, se utilizan dos métricas de análisis: interacción y porcentaje de engagement (entendiendo por tal el nivel de implicación, compromiso, o fidelidad de la audiencia) que logran los mensajes difundidos en las redes sociales analizadas.

Las conclusiones del estudio sugieren que la marca política Vox es la que logra mayor visibilidad e impacto en redes sociales en España. Con respecto al uso de redes sociales, en el ámbito de la comunicación política, Instagram se consolida como la de mayor crecimiento y la que mayor viralidad logra dar a los mensajes analizados. Sin embargo, todavía no es la preferida por los partidos más tradicionales, tal y como lo muestra el perfil bajo de actividad que mantienen en esta red.

Palabras clave: comunicación política, redes sociales, partidos políticos; España.

\begin{abstract}
Social networks play an important role in the dissemination of messages, not only during electoral periods, but also in any political communication strategy. The objective of this work is to analyze the communication of the main Spanish political parties in social networks from the point of view of reception studies. For this, the audience's response to the messages launched by political parties during a month, in the four main social networks (Twitter, Facebook, YouTube and Instagram) is analyzed. To this end, two analysis metrics are used: interaction and percentage of engagement (understanding the level of involvement, commitment, or loyalty of the audience) that achieve the messages disseminated in the social networks analyzed.

The conclusions of the study suggest that the Vox political brand is the one that achieves greater visibility and impact on social networks in Spain. With respect to the use of social networks, in the field of political communication, Instagram is consolidated as the one with the highest growth and the one that achieves the highest virality in the messages analyzed. However, it is not yet preferred by the more traditional parties, as shown by the low activity profile they maintain in this network.
\end{abstract}

Keywords: communication, media, political communication, social networks, political parties; Spain.

Recibido: 2019-10-09. Aceptado: 2019-12-13. ID: 6332.http://dx.doi.org/10.15304/marco.5.6332 


\section{INTRODUCCIÓN}

\subsection{Objetivos}

El objetivo de este trabajo es identificar las estrategias de contenido más virales y eficaces de los partidos políticos en España, mediante el análisis de la visibilidad y tráfico en redes sociales, a partir de la interacción y el nivel de engagement que presentan las cuentas de las distintas formaciones políticas. El diseño metodológico tiene como objetivo responder a las siguientes preguntas de investigación:

1. H1. ¿Cuáles son los mensajes en redes sociales lanzados por partidos políticos españoles qué logran mayor nivel de interacción y engagement?

2. H2. ¿Qué nivel de visibilidad y repercusión de sus mensajes tienen los distintos partidos políticos en las redes sociales?

3. H3. ¿Cuentan los partidos políticos con una estrategia diseñada para las distintas redes sociales?

\subsection{Justificación}

El uso de redes sociales se ha extendido de una forma rápida. En España, el $85 \%$ de los internautas de 16-65 años utilizan redes sociales, lo que representa más de 25,5 millones de personas. Teniendo en cuenta que la población usuaria de redes de promedio está presente en 3,7 redes, según el Estudio Anual de Redes 2019, elaborado por IAB, Facebook presenta un $87 \%$ de uso, seguida de Youtube (68\%), Instagram (54\%) y Twitter (50\%). Con respecto a la actividad de los usuarios de redes sociales, el uso principal de las redes es social (chatear, enviar mensajes, etc...); sin embargo, un 30\% afirma que "muy o bastante frecuentemente" siguen cuentas de partidos políticos.

Los datos de uso muestran que Internet es una plataforma estratégica de comunicación para los partidos políticos (Congosto, 2015). Y no solo Internet, las redes sociales se han integrado como una vía más de distribución de mensajes en la campaña electoral, hasta el punto que constituyen una forma de comunicación política (Skogerbø 2011) que, como añade Ruano, López \& Mosquera (2018), funciona como como "espacio de debate fundamental para entender los procesos electorales contemporáneos". Las redes sociales modifican los procesos tradicionales de configuración de la opinión pública y permiten comunicarse con los electores y difundir sus programas electorales; de forma que se genera un proceso comunicativo bidireccional que modifica los flujos participativos en política (Alonso, 2016:96).

Los mensajes y la conversación que se producen en esa nueva esfera política virtual contribuyen a la construcción del mensaje político. Todo ello supone un desafío, pues se requieren de nuevas aproximaciones y métodos para medir los efectos de los mensajes políticos en las redes sociales.

La política y sus actores encuentran numerosas razones para incorporar las redes sociales en sus procesos comunicativos. En primer lugar, permiten aumentar la visibilidad de 
sus candidaturas y partidos en la esfera pública; además, son especialmente útiles para llegar a determinados grupos de votantes, por ejemplo, los jóvenes; $y$, por último, permiten amplificar la estrategia de comunicación incorporando nuevas vías de distribución de sus mensajes, también con el objetivo de que lleguen a medios de comunicación y periodistas. Por todo ello, cada vez es más aceptado un uso de las redes sociales para la comunicación política, no solo en momentos puntuales de procesos electorales, sino de forma coordinada y coherente con el programa y el plan de comunicación del grupo político.

\subsection{Marco teórico}

El objetivo de este trabajo es detectar las estrategias de contenido más virales y eficaces de los partidos políticos en España, mediante el análisis de la visibilidad y tráfico en redes sociales. Para ello se analiza la interacción de las distintas formaciones en las cuatro principales redes sociales y el nivel de interacción que presentan los distintos perfiles. El concepto engagement es un galicismo que tiene distintas acepciones y que en el contexto de este trabajo puede traducirse como nivel de implicación, compromiso, o fidelidad de la audiencia con respecto a un determinado contenido o post, distribuido a través de redes sociales. Para la medición del nivel de engagement, tal y como se propone en la metodología de este trabajo, se utiliza una métrica de redes sociales.

En otros trabajos se analizan cuáles son las estrategias de comunicación política en las redes sociales, a partir del estudio del contenido de los mensajes difundidos y de la estrategia, patrón de publicación o recursos que se utilizan en los mensajes publicados en perfiles (posts) de candidatos y partidos. Diversos estudios han analizado también cómo las redes fomentan el debate que surge con la difusión de esos mensajes, considerando las redes una nueva esfera digital o, como señala Alonso (2016, p.97), ágora digital para el debate público. En esa misma línea, pero aportando conclusiones que complementan estos enfoques, se referencian estudios en los que se subraya cómo las redes sociales reproducen rasgos de la comunicación política, como la repetición de eslóganes y la comunicación política unidireccional (Amado \& Tarullo, 2015; Moya Sánchez \& Herrera Damas, 2015); así como también la reproducción de actitudes de personalización en política, aunque esta en las redes adquiera una mayor viralización fruto de la participación de la audiencia (Casero-Ripollés, 2015; Pérez-Curiel \& García-Gordillo, 2018).

Se han realizado distintos estudios sobre la comunicación de los principales partidos políticos españoles en las redes sociales desde el punto de vista de los estudios de recepción. La mayor parte de ellos se centran en momentos próximos a contiendas electorales. Este es el caso del trabajo de Congosto (2015) y también de Ramos, Fernández y Pineda (2018), que analiza la viralidad de mensajes publicados en Twitter durante la campaña electoral de las elecciones europeas. Mientras que escasean los estudios comparativos de engagement en las distintas redes, numerosos autores se centran en análisis circunscritos a una red, como Youtube (Gulati y Williams 2010; May, 2008; Berrocal-Gonzalo, Martín-Jiménez y Gil-Torres, 2017); Twitter (Criado, Martinez y Silván, 2012; Zugasti-Azagra, y Pérez González, 2015; Jivkova-Semova, Requeijo-Rey y Padilla-Castillo, 2017); Facebook (Abejón-Mendoza y Mayoral-Sánchez, 2017) o Instagram (Selva-Ruiz y Caro-Castaño, 2017; Quevedo-Redondo y Portalés-Oliva, 2017). 
También son numerosos los análisis de campañas electorales en países, como Colombia (Ruano, López y Mosquera, 2018); Chile (López-Hermida y Fierro-Zamora, 2016), Suecia (Larsson y Hallvar, 2010) o Alemania (Tumasjan, Sprenger, Sandner y Welp, 2012). Otros trabajos establecen correlación entre el tipo de comunicación que desarrollan los partidos políticos a través de los medios sociales y la orientación ideológica (Said-Hung, Prati y Cancino-Borbón, 2017) e incluso con la intención de voto de los ciudadanos (Alonso, 2016). En esta línea, Tumasjan, Sprenger, Sandner y Welp (2012) demuestran en sus estudios que el número de usuarios de redes sociales y los mensajes explican la tendencia de voto.

Las redes sociales permiten poner el foco en el candidato individual más que en el partido político, expandiendo así la arena política a las posibilidades de campañas personalizadas (Zittel, 2009). Enli y Skogerbø (2013) analizaron las estrategias y prácticas en las redes sociales de los políticos noruegos en Facebook y Twitter y concluyeron que el uso de las redes sociales por parte de los líderes políticos logra la visibilidad personal de candidaturas, por lo que las redes sociales se han convertido en una nueva herramienta para la política personal, pero no funcionan, al menos por el momento, como una parte completamente establecida y coordinada de la comunicación de la campaña.

Muchos de los estudios utilizan el análisis de contenido de los mensajes en redes sociales durante campañas políticas. Este es el caso de Ruano, López y Mosquera (2018) que monitorizan a cuatro candidatos para analizar el contenido temático de la participación; y de Gómez-Calderón, Roses y Paniagua-Rojano (2017) que aplican un análisis de contenido de los mensajes en Twitter de los cuatro líderes políticos en España (PP, PSOE, Ciudadanos y Podemos), detectando ciertos patrones de uso que difieren de sus homólogos en otros países. Santos y Gomes (2017) también aplican un análisis de contenido de los mensajes en redes sociales de 512 congresistas en Brasil, comparando diversa información sobre el perfil y uso de la cuenta (fecha de creación; número total de seguidores; número total de perfiles que siguen; mensajes publicados, etc...). Los datos de los perfiles fueron cruzados con las distintas ideologías y partidos, concluyendo que los candidatos, y consecuentemente partidos, más próximos a la izquierda en Brasil hacen un uso más intensivo de las redes sociales.

En los estudios antes referidos se observa que la visibilización de problemáticas en redes sociales viene a coincidir con los temas habituales de la agenda pública, aunque en las redes sociales la exposición de argumentos adquiere matices más propios de un debate, que de un discurso. Las corrientes de opinión tienden a posicionarse de igual forma que en la arena pública, aunque el efecto cascada de tuits y retuits que permiten las redes sociales tiende a amplificar algunos debates. Un elemento diferencial que se observa en el debate político en redes sociales es la posibilidad de que la voz del emisor, en este caso partido político o candidato, pueda mantenerse y seguir participando de ese debate, modulando en cierta medida ese proceso de viralización que puede darse en las redes. Para ello, los debates pueden seguir una forma estructurada y jerarquizada, normalmente mediante el uso de \#hahstags; o adoptar una forma más horizontal e interactiva, mediante las interacciones de compartición (replys, retuits, etc...) que ofrecen las redes sociales a los usuarios.

Campos-Domínguez y García-Orosa (2018) subrayan como elemento de análisis de 
los mensajes políticos en redes la importancia del uso de algoritmos y datos. La denominada comunicación algorítmica forma parte, aunque de forma incipiente, de la estrategia de todos los partidos con representación parlamentaria en España. En las entrevistas que realizaron los autores referidos a los responsables de campaña de las generales de 2015, todos negaron la existencia de bots gestionados desde la coordinación del partido para la generación de mensajes, aunque admitieron la existencia de colaboradores externos que, a modo de voluntarios, dinamizan otros perfiles próximos a las cuentas de los partidos y con los que comparten argumentos, marcos y temas favorables al partido. Por el contrario, el uso de bots es habitual para la segmentación de mensajes, la medición de impactos y el seguimiento de la actividad en redes de otros partidos políticos (Campos-Domínguez y García-Orosa, 2018).

Asimismo, se observa una relación entre un nivel alto de engagement en redes sociales por parte de la audiencia, con una alta intención de voto. De ahí que numerosos análisis, como el de Alonso (2016) o Santos y Gomes (2017) se centren en el nivel de engagement medido a través de distintas métricas, tales como número de seguidores, tweets totales, cuentas seguidas, listas, favoritos, retuits, respuestas y enlaces multimedia.

Por su parte, Hernández-Santaolalla y Sola-Morales (2019) ahondan en la importancia del engagement y analizan su relación con el recurso de la postverdad, centrando el análisis en mensajes de Twitter durante periodo electoral, lo que les permite concluir a los autores que se observa una tendencia de los políticos a seguir estrategias retóricas basadas en la emoción y los sentimientos proactivos; mientras que por parte de los usuarios, se observa, con respecto a los políticos, una mayor inclinación a la defensa a ultranza de sus argumentos.

\section{MATERIAL Y MÉTODOS}

Con el objetivo de dar respuesta a las cuestiones planteadas en relación al objetivo de investigación, se monitoriza durante un mes (del 10.08.2019 al 10.09.2019) los tuits que logran más interacciones y engagement en las cuatro redes sociales más utilizadas (Facebook, Twitter, Youtube e Instagram).

De acuerdo al objetivo de investigación, se diseña un método cuantitativo, que permite recabar datos de monitoreo de la participación de los partidos políticos y candidatos en redes sociales.

Para testear el método, se diseña una prueba piloto que consiste en la monitorización durante una semana de los post en las distintas redes sociales que logran mayor viralidad, medida esta a partir del nivel de interacción y engagement en las redes sociales analizadas, tanto por parte de políticos, como de candidatos.

Para el análisis de datos, se utiliza la herramienta digital scanner, del software Icuarus (Epsilon Technologies), que utiliza un panel de análisis de más de 7000 perfiles. Para la validación de la herramienta de análisis y comprobar el funcionamiento de la ficha de recogida de datos, se realiza un análisis exploratorio de los resultados en una muestra de 7 días (periodo de 29 de julio al 4 de agosto de 2019). 
Una vez validada la herramienta metodológica, se aplica el análisis a una muestra de 30 días (periodo del 10 de agosto al 10 de septiembre de 2019). La muestra de tiempo analizado comprende un periodo que abarca una primera etapa de baja intensidad informativa durante agosto, hasta la primera parte de septiembre que comprende el inicio del curso político. Entre las noticias más relevante de la actualidad durante el periodo analizado está la desaparición, búsqueda y posterior aparición del cadáver de Blanca Fernández Ochoa; los efectos devastadores de el huracán Dorian y su acercamiento a la costa de Estados Unidos; la muerte de Camilo Sesto; la aprobación del Parlamento británico de la ley que exigiría a Johnson un Brexit pactado y en la arena política española, la imputación a Aguirre y Cifuentes por corrupción en el 'caso Púnica' y el reinicio de la actividad política con la reunión entre PSOE y Unidas Podemos para retomar las conversaciones con el objetivo de la búsqueda de un acuerdo de gobierno que evitara una repetición electoral.

Algunos trabajos centran su objetivo de estudio en evaluar el impacto de los mensajes en las redes. Para ello, junto con el concepto de viralidad, se ha venido utilizando algunos otros, como stickiness o engagement, referidos a métricas que miden los niveles de involucración por parte de los usuarios con el contenido de un determinado post en una red social. Para medir ese nivel de engagement algunos autores han utilizado fórmulas de métricas existentes. Alonso (2016) desarrolla una matriz de análisis que incluye diez indicadores (presencia, creación, seguidores, posts publicados, media diaria, compartidos, enlaces, comentarios y 'me gusta') agrupados en tres bloques: presencia y potencial de influencia, actividad e interacción y engagement. Con esa misma finalidad de ponderar tanto el número de usuarios, como la interacción y debate que generan los mensajes, Moya-Sánchez y Herrera-Damas (2016) analizan el comportamiento del dirigente político en redes sociales, con la finalidad de evaluar su potencial persuasivo, mediante el estudio de la tipología y frecuencia de sus mensajes. Para ello, crean el índice global de potencial persuasivo (IGPP), que se obtiene de las variables de número de usuarios y peso comunicacional de los distintos tipos de mensajes emitidos, la frecuencia de emisión y la amplificación que encuentran en la red de seguidores (Moya y Herrera, 2016).

De acuerdo al objetivo de este trabajo, se propone utilizar aquí dos métricas que permiten evaluar el efecto que en la audiencia tiene un contenido o mensaje, que en las redes adopta la forma de post, tras su difusión en redes. Las dos métricas que se utilizan en este trabajo son número total de interacciones y porcentaje de engagement. Hay que considerar que cada red social tiene unas reglas de funcionamiento, por lo que la reacción del usuario ante un determinado mensaje se mide de forma distinta. En este trabajo, se sigue el modelo ya utilizado en sus análisis por el Observatorio sectorial de Redes sociales de IAB. En la siguiente tabla se identifican los conceptos medibles para evaluar el nivel de interacción y de engagement para cada una de las redes.

Para la medición de la interacción de un mensaje, indistintamente de la red social (Facebook, Twitter, Youtube e Instagram) se considerará el número de reacciones que dicho post ha generado en la red social correspondiente; mientras que para el nivel de engagement se considerará tanto el número de "me gusta" (likes), como el de seguidores, en relación al número total de interacciones. Para la medición del nivel de engagement otros autores esta- 
blecen fórmulas homólogas, tales como la suma de los favoritos y de los retweets de cada tweet, dividido entre el número de seguidores del perfil (Herrera-Torres et al., 2017; Carrasco-Polaino, Villar-Cirujano y Martín-Cárdaba, 2019).

En la siguiente tabla puede observarse la homologación de conceptos entre las cuatro redes sociales analizadas, así como la forma de obtenerse las dos métricas que se utilizan en este estudio.

Tabla 1. Cómo calcular número total de interacciones y porcentaje de engagement en las cuatro redes sociales (Facebook, Twitter, Youtube, Instagram)

\begin{tabular}{|l|c|c|c|c|}
\hline $\begin{array}{l}\text { Número total de } \\
\text { interacciones }\end{array}$ & $\begin{array}{c}\text { Facebook } \\
\text { (likes }+ \\
\text { comments } \\
\text { shares })\end{array}$ & $\begin{array}{c}\text { Twitter } \\
\text { (favs }+ \\
\text { menciones }+ \\
\text { retweet) }\end{array}$ & $\begin{array}{c}\text { Youtube } \\
\text { (likes }+ \text { dislikes } \\
+ \text { comments) }\end{array}$ & $\begin{array}{c}\text { Instagram } \\
\text { comments } \\
+ \text { saves })\end{array}$ \\
\hline $\begin{array}{l}\text { Porcentaje de } \\
\text { engagement }\end{array}$ & \multicolumn{3}{|c|}{ [(likes+seguidores)/interacciones)] } \\
\hline
\end{tabular}

Fuente: elaboración propia

\section{ANÁLISIS DE LOS RESULTADOS}

Los datos de uso de redes sociales muestran la importancia creciente de la comunidad de usuarios y, a su vez, del uso que hacen estos para consultar noticias de actualidad y seguir la información de partidos políticos y líderes. Por este motivo, los partidos y formaciones destinan recursos para mantener perfiles y cuentas activas. En la siguiente tabla, puede observarse el número de seguidores de cada una de las principales formaciones políticas.

Tabla 2. Número de seguidores /suscriptores de las cuentas de los partidos políticos.

\begin{tabular}{|l|c|c|c|c|}
\hline & Facebook & Twitter & Youtube & Instagram \\
\hline Partido Popular & 205.290 & 7.189 .000 & 17.607 & 77.236 \\
\hline Partido Socialista & 185.133 & 7.019 .000 & 18.384 & 74.925 \\
\hline Ciudadanos & 336.663 & 5.208 .000 & 46.198 & 101.967 \\
\hline Podemos & 191.651 & 1.400 .000 & 84.484 & 175.847 \\
\hline Vox & 347.888 & 2.856 .000 & 185.000 & 340.763 \\
\hline
\end{tabular}

Fuente: elaboración propia

De acuerdo a la premisa de que no sólo es importante el número de seguidores de una cuenta, sino que también lo es el comportamiento y reacción de estos ante los mensajes, en este trabajo se analiza el nivel de interacción y engagement de esos perfiles. El estudio se basa en la actividad de esas cuentas en redes sociales (Facebook, Instagram, Twitter y YouTube). En el ranking de los veinte posts que logran los niveles más altos se observa que todos fueron 
lanzados desde las cuentas de partidos políticos y ninguno desde perfiles de candidatos. La conclusión es homóloga, a tenor de los datos de porcentaje de nivel de engagement.

Asimismo, se observa que de acuerdo (a) al número total de interacciones, es el partido VOX el que logra colocar mayor número de mensajes entre los posts con mayor nivel de interacción. Hasta el punto de que, durante el mes analizado, los veinte posts con mayor nivel de interacción correspondieron todos a esta formación política. Asimismo, (b) se observa que es la red Instagram en la que, seguida de Facebook, los mensajes logran un mayor nivel de interacción. Los mensajes mejor posicionados en el ranking que tuvieron su origen en Twitter y Youtube se sitúan en las últimas posiciones de la tabla (puesto 14 y 19, respectivamente).

Tabla 3. Los veinte mensajes de partidos políticos españoles en redes sociales con mayor nivel de interacción.

\begin{tabular}{|c|c|c|c|c|c|}
\hline Ranking & Perfil & Interac. & $\%$ Eng. & Red social & POST-URL \\
\hline 1 & VOX & 45.115 & 13,66 & Instagram & $\begin{array}{l}\text { Explusión de los ilegales YA! } \\
\square \text { https://www.instagram.com/p/B1yCAhyo10P/ }\end{array}$ \\
\hline 2 & VOX & 43.645 & 13,30 & Instagram & $\begin{array}{l}\text { VOX denunciará al Open Arms por favorecer la } \\
\text { inmigración ilegal } \\
\square \text { https://www.instagram.com/p/B1bB1BCoDRn/ }\end{array}$ \\
\hline 3 & VOX & 41.927 & 13,38 & Facebook & $\begin{array}{l}\text { Grande Juan Carlos } \\
\square \text { https://www.facebook.com/467127060059387/ } \\
\text { posts/2076942415744502 }\end{array}$ \\
\hline 4 & VOX & 41.015 & 12,45 & Instagram & $\begin{array}{l}\text { "Dibujar la bandera española en el cielo es lo más } \\
\text { grande". } \\
\square \text { https://www.instagram.com/p/B1 owtF3Ba2y/ }\end{array}$ \\
\hline 5 & VOX & 39.310 & 11,86 & Instagram & $\begin{array}{l}\text { Quién paga el pato? La factura la pagan los } \\
\text { españoles de a pie. } \\
\square \text { https://www.instagram.com/p/B13jKGAos1c/ }\end{array}$ \\
\hline 6 & VOX & 38.534 & 11,60 & Instagram & $\begin{array}{l}\text { Respeto y convivencia. La lógica, tarde o temprano, } \\
\text { se impondrá. } \square \mathrm{https} / / \text { www.instagram.com/p/ } \\
\text { B16Gu90ICUv/ }\end{array}$ \\
\hline 7 & VOX & 35.286 & 11,36 & Facebook & $\begin{array}{l}\text { Asi ha quedado el puesto de la Guardia Civil en } \\
\text { Ceuta tras el salto de más de } 150 \text { inmigrantes esta } \\
\text { madrugada. } \\
\square \text { https://www.facebook.com/467127060059387/ } \\
\text { posts/2068493849922692 }\end{array}$ \\
\hline 8 & VOX & 34.731 & 11,21 & Facebook & $\begin{array}{l}\text { Santiago Abascal dice verdades como puños en } \\
\text { la tribuna del Congreso sobre el \#OpenMafia, } \\
\text { inmigración } \\
\square \text { https://www.facebook.com/467127060059387/ } \\
\text { posts/421189175179298 }\end{array}$ \\
\hline 9 & VOX & 33.636 & 10,86 & Facebook & $\begin{array}{l}\text { DIRECTO Santiago Abascal interviene en el } \\
\text { Congreso sobre el Open Arms. } \\
\square \text { https://www.facebook.com/467127060059387/ } \\
\text { posts/2067034700068607 }\end{array}$ \\
\hline 10 & VOX & 32.593 & 9,790 & Instagram & $\begin{array}{l}\text { @ivanedlm desmonta el consenso progresista en } \\
\text { torno a la inmigración ilegal. } \\
\square \text { https://www.instagram.com/p/B18vtFqoVxx/ }\end{array}$ \\
\hline
\end{tabular}




\begin{tabular}{|c|c|c|c|c|c|}
\hline 11 & VOX & 32.197 & 10,30 & Facebook & $\begin{array}{l}\text { VOX no acepta inmigrantes ilegales. } \\
\square \text { https://www.facebook.com/467127060059387/ } \\
\text { posts/2075199429252134 }\end{array}$ \\
\hline 12 & VOX & 31.395 & 9,260 & Instagram & $\begin{array}{l}\text { Se cumplen dos años del pleno infame en el } \\
\text { Parlamento de Cataluña } \\
\square \text { https://www.instagram.com/p/B2HTRw5o7BB/ }\end{array}$ \\
\hline 13 & VOX & 29.896 & 9,040 & Instagram & $\begin{array}{l}\text { Desde luego es verosímil, pero ¿creéis que es real? } \\
\square \text { https://www.instagram.com/p/B10-DSHhrPQ/ }\end{array}$ \\
\hline 14 & VOX & 29.867 & 0,11 & Twitter & $\begin{array}{l}\text { Nadie mejor que alguien del campo, que trabaja } \\
\text { los siete días de la semana con los animales, para } \\
\text { desmentir las chorradas de los falsos ecologistas } \\
\square \text { https://twitter.com/vox_es/ } \\
\text { status/1168574325290688513 }\end{array}$ \\
\hline 15 & VOX & 29.348 & 8,90 & Instagram & $\begin{array}{l}\text { Joven diputado de VOX fulmina a los diputados } \\
\text { separatistas } \\
\square \text { https://www.instagram.com/p/B1qth6CBpwd/ }\end{array}$ \\
\hline 16 & VOX & 28.889 & 8,82 & Instagram & $\begin{array}{l}\text { Revuelta patriótica en Salou. } \\
\square \text { https://www.instagram.com/p/B1UX3mAhqjp/ }\end{array}$ \\
\hline 17 & VOX & 26.844 & 8,25 & Instagram & $\begin{array}{l}\text { El futuro, tan europeo y cosmopolita, era esto. } \\
\square \text { https://www.instagram.com/p/B1CKEONheGM/ }\end{array}$ \\
\hline 18 & VOX & 25.832 & 8,29 & Facebook & $\begin{array}{l}\text { Santiago Abascal "¿Quién paga el pato? } \\
\square \text { https://www.facebook.com/467127060059387/ } \\
\text { posts/2071827682922642 }\end{array}$ \\
\hline 19 & VOX & 23.671 & 13,23 & Youtube & $\begin{array}{l}\text { Colosal réplica de Santiago Abascal en la tribuna } \\
\text { del Congreso } \\
\square \text { https://www.youtube.com/ } \\
\text { watch?v=_0OLUW7SpfQ }\end{array}$ \\
\hline 20 & VOX & 23.545 & 7,02 & Instagram & $\begin{array}{l}\text { Nunca dejaremos de decirlo: urge iniciar la } \\
\text { repatriación inmediata de todo inmigrante.... } \\
\square \text { https://www.instagram.com/p/B2B54FuBwtZ/ }\end{array}$ \\
\hline
\end{tabular}

Fuente: elaboración propia.

Por otra parte, de acuerdo al (b) porcentaje de engagement que logran los posts, se observa como, aunque los mensajes de VOX sigue ocupando la mayor parte de las posiciones del ranking, es el Partido Popular el que logra posicionar su contenido con el mejor nivel gracias al uso de Youtube. Sin embargo, es VOX el partido que logra en términos generales un mejor nivel de engagement con sus contenidos en redes sociales, ya que de los 20 contenidos mejor posicionados, tan solo dos (ocupando el primer y décimo lugar) pertenecen al Partido Popular y el resto a VOX. Cabe destacar que estos dos mensajes del Partido Popular que aparecen en posiciones elevadas de la tabla son contenidos difundidos en la red Youtube, y que se corresponden con la primera intervención de Cayetana Álvarez de Toledo como portavoz en el pleno extraordinario del Congreso.

El resto de contenidos se corresponden por tanto con mensajes de VOX en redes sociales y preferentemente, al igual que en la métrica de interacciones difundidos, a través de la red Instagram, aunque también aparece en el ranking otros posts lanzados desde Facebook y Youtube. Nuevamente, Twitter es la gran ausente, lo que confirma que es la red social en la que los partidos encuentran más dificultades para lograr un nivel alto de interacciones y engagement en sus contenidos. 
Tabla 4. Los veinte mensajes de partidos políticos españoles en redes sociales con mayor nivel de engagement.

\begin{tabular}{|c|c|c|c|c|c|}
\hline Ranking & Perfil & Interac. & $\%$ Eng. & Red social & POST-URL \\
\hline 1 & PP & 14,91 & 2.506 & Youtube & $\begin{array}{l}\text { Segunda intervención de Cayetana Álvarez de Toledo } \\
\text { en el pleno extraordinario del Congreso } \square \text { https:// } \\
\text { www.youtube.com/watch?v=xrq78HfCPaQ }\end{array}$ \\
\hline 2 & VOX & 13,66 & 45.115 & Instagram & $\begin{array}{l}\text { ¡Expulsión de los ilegales ya! } \\
\square \text { https://www.instagram.com/p/B1yCAhyo10P/ }\end{array}$ \\
\hline 3 & VOX & 13,38 & 41.927 & Facebook & $\begin{array}{l}\text { ¡Grande Juan Carlos!Así deja en ridículo a las falsas } \\
\text { ecologistas en pleno directo } \\
\square \text { https://www.facebook.com/467127060059387/ } \\
\text { posts/2076942415744502 }\end{array}$ \\
\hline 4 & VOX & 13,30 & 43.645 & Instagram & $\begin{array}{l}\text { VOX denunciará al Open Arms por favorecer la } \\
\text { inmigración ilegal } \\
\square \text { https://www.instagram.com/p/B1bB1BCoDRn/ }\end{array}$ \\
\hline 5 & VOX & 13,23 & 23.671 & Youtube & $\begin{array}{l}\text { Colosal réplica de Santiago Abascal en la tribuna } \\
\text { del Congreso } \square \text { https://www.youtube.com/ } \\
\text { watch?v=_0OLUW7SpfQ }\end{array}$ \\
\hline 6 & VOX & 12,45 & 41.015 & Instagram & $\begin{array}{l}\text { Recordamos las palabras de Francisco Marín Núñez } \\
\text { "Dibujar la bandera española en el cielo es lo más } \\
\text { grande" } \\
\square \text { https://www.instagram.com/p/B1owtF3Ba2y/ }\end{array}$ \\
\hline 7 & VOX & 11,88 & 21.255 & Youtube & $\begin{array}{l}\text { Santiago Abascal destruye con argumentos al Open } \\
\text { Arms y al felón de Sánchez } \\
\square \text { https://www.youtube.com/ } \\
\text { watch?v=HoMAoUI8mlE }\end{array}$ \\
\hline 8 & VOX & 11,86 & 39.310 & Instagram & $\begin{array}{l}\text { @santi_abascal "¿Quién paga el pato? } \\
\square \text { https://www.instagram.com/p/B13jKGAos1c/ }\end{array}$ \\
\hline 9 & VOX & 11,06 & 38.534 & Instagram & $\begin{array}{l}\text { Respeto y convivencia. La lógica, tarde o temprano, } \\
\text { se impondrá. } \\
\square \text { https://www.instagram.com/p/B16Gu90ICUv/ }\end{array}$ \\
\hline 10 & PP & 11,57 & 1.921 & Youtube & $\begin{array}{l}\text { Cayetana Álvarez de Toledo: "El sanchismo y el } \\
\text { salvinismo son las dos caras de la misma moneda". } \\
\square \text { https://www.youtube.com/watch?v=B7kcv2SOQy0 }\end{array}$ \\
\hline 11 & VOX & 11,36 & 35.286 & Facebook & $\begin{array}{l}\text { Así ha quedado el puesto de la Guardia Civil en } \\
\text { Ceuta tras el salto de más de } 150 \text { inmigrantes } \\
\square \text { https://www.facebook.com/467127060059387/ } \\
\text { posts/2068493849922692 }\end{array}$ \\
\hline 12 & VOX & 11,21 & 34.731 & Facebook & $\begin{array}{l}\text { DIRECTO Santiago Abascal interviene en el } \\
\text { Congreso sobre el Open Arms. } \\
\square \text { https://www.facebook.com/467127060059387/ } \\
\text { posts/421189175179298 }\end{array}$ \\
\hline 13 & VOX & 10,86 & 33.636 & Facebook & $\begin{array}{l}\text { Santiago Abascal dice verdades como puños en } \\
\text { la tribuna del Congreso sobre el \#OpenMafia, } \\
\text { inmigración e inseguridad } \\
\square \text { https://www.facebook.com/467127060059387/ } \\
\text { posts/2067034700068607 }\end{array}$ \\
\hline 14 & VOX & 10,30 & 32.197 & Facebook & $\begin{array}{l}\text { Ivan Espinosa de los Monteros desmonta el consenso } \\
\text { progresista en torno a la inmigración ilegal. } \\
\square \text { https://www.facebook.com/467127060059387/ } \\
\text { posts/2075199429252134 }\end{array}$ \\
\hline 15 & VOX & 9,80 & 17.777 & Youtube & $\begin{array}{l}\text { El vídeo que los progres y las mafias de la } \\
\text { inmigración no quieren que veas } \\
\square \text { https://www.youtube.com/watch?v=HdWUrqSey94 }\end{array}$ \\
\hline 16 & VOX & 9,79 & 32.593 & Instagram & $\begin{array}{l}\text { @ivanedlm desmonta el consenso progresista en } \\
\text { torno a la inmigración ilegal. } \\
\square \text { https://www.instagram.com/p/B18vtFqoVxx/ }\end{array}$ \\
\hline
\end{tabular}




\begin{tabular}{|c|c|c|c|c|l|}
\hline 17 & VOX & 9,26 & 31.395 & Instagram & $\begin{array}{l}\text { Se cumplen dos años del pleno infame en el } \\
\text { Parlamento de Cataluña } \\
\square \text { https://www.instagram.com/p/B2HTRw5o7BB/ }\end{array}$ \\
\hline 18 & VOX & 9,04 & 29.896 & Instagram & $\begin{array}{l}\text { Se está moviendo mucho este vídeo... Desde luego es } \\
\text { verosímil, pero ¿creéis que es real } \\
\square \text { https://www.instagram.com/p/B10-DSHhrPQ/ }\end{array}$ \\
\hline 19 & VOX & 8,90 & 29.348 & Instagram & $\begin{array}{l}\text { Joven diputado de VOX fulmina a los diputados } \\
\text { separatistas } \\
\square \text { https://www.instagram.com/p/B1qth6CBpwd/ }\end{array}$ \\
\hline 20 & VOX & 8,82 & 28.889 & $\begin{array}{l}\text { Revuelta patriótica en Salou. Militantes de VOX } \\
\text { resisten y frenan una manifestación de los separatistas } \\
\text { https://www.instagram.com/p/B1UX3mAhqjp/ }\end{array}$ \\
\hline
\end{tabular}

Fuente: elaboración propia

Del análisis comparado por resultados en redes (Facebook, Twitter, Youtube e Instagram), tanto en lo referente al nivel de interacción, como de engagement, se observa que VOX presenta, con respecto al resto de formaciones políticas, una estrategia acertada de comunicación en la red Instagram y también, aunque en menor medida, en Twitter y Facebook, ya que comparte el liderato de audiencia con la formación Podemos, que logra colocar colar en el ranking algunos mensajes en Twitter (segundo lugar) y Facebook (octava posición).

Singular interés reviste la estrategia de comunicación del PP en Youtube en la que excepcionalmente una selección muy precisa de contenidos les genera un alto nivel de interacciones (puesto primero y cuarto en el ranking). Por último, señalar que los tipos de contenido que se utilizan en los posts que logran el mayor nivel de interacción y engagement son videos, seguidos de fotografías.

Tabla 5. Posiciones ocupadas por los partidos políticos españoles en el top ten delas cuatro redes sociales con mayor nivel de interacción y engagement.

\begin{tabular}{|c|c|c|c|c|c|c|c|c|}
\hline & \multicolumn{4}{|c|}{ INTERACCIONES } & \multicolumn{4}{|c|}{$\%$ ENGAGEMENT } \\
\hline & Instagram & Facebook & Twitter & Youtube & Instagram & Facebook & Twitter & Youtube \\
\hline 1 & VOX & VOX & VOX & VOX & VOX & VOX & VOX & PP \\
\hline 2 & VOX & VOX & Podemos & VOX & VOX & VOX & VOX & VOX \\
\hline 3 & VOX & VOX & VOX & VOX & VOX & VOX & VOX & VOX \\
\hline 4 & VOX & VOX & VOX & Podemos & VOX & VOX & VOX & PP \\
\hline 5 & VOX & VOX & VOX & PP & VOX & VOX & VOX & VOX \\
\hline 6 & VOX & VOX & VOX & Ciudadanos & VOX & VOX & VOX & VOX \\
\hline 7 & VOX & VOX & VOX & PSOE & VOX & VOX & VOX & VOX \\
\hline 8 & VOX & Podemos & VOX & PP & VOX & VOX & VOX & VOX \\
\hline 9 & VOX & VOX & VOX & $\mathrm{PP}$ & VOX & VOX & VOX & VOX \\
\hline 10 & VOX & VOX & VOX & PP & VOX & VOX & VOX & VOX \\
\hline
\end{tabular}

En conclusión, del análisis de la estrategia de publicación y de los flujos de mensajes en redes sociales de los principales partidos políticos, se observa que parte del éxito de Vox 
se debe a las colaboraciones con otras cuentas en redes, especialmente cuando estas son de perfiles prescriptores, esto es, cuentas con un alto nivel de seguidores y actividad. Tal y como se observa en el perfil de Vox, este cuenta con seguidores que actúan como amplificadores coordinados del mensaje (por ejemplo, las cuentas de Santiago Abascal, Ricardo Chamorro, simpatizantes Vox, etc...). Esta es una manera eficiente de incrementar el nivel de interacción de los mensajes.

La segunda estrategia que se observa en Vox es que tiene presente las características del público al que se dirigen, esto es, un público joven y usuario habitual de redes sociales. Centran el desarrollo de sus mensajes de ideas fuerza en el target objetivo Por ello, adaptan el mensaje a la audiencia, utilizando recursos característicos de las redes sociales: lanzan mensajes sencillos, incorporan en la mayoría vídeo o imágenes, incluyen hastags, etc... Asimismo, utilizan gifs, memes y emoticonos, como recursos para establecer un vínculo con la audiencia, muchas veces rozando lo burdo, al simplificar el mensaje a lo más puramente emocional.

La tercera estrategia observada es que la mayor parte de sus contenidos están vinculados a hashtags relacionados con tendencias de actualidad y temas de interés para la audiencia, o bien noticias del día, programas de televisión; o en otras ocasiones, es simplemente una idea fuerza del argumentario que se repite a través de distintos líderes y en los distintos medios de comunicación.

\section{DISCUSION Y CONCLUSIONES}

Las redes sociales, como en su día ocurriesen con Internet, se integran en el mix de la estrategia de comunicación de los partidos políticos. Sin embargo, se trata de un fenómeno tan reciente que todavía los emisores exploran las distintas posibilidades de hacer llegar el mensaje a su público con mayor eficacia.

Hasta ahora, diversos estudios han analizado la presencia de partidos y líderes en redes sociales y, asimismo, han aplicado análisis de contenido para determinar la influencia de las agendas (prioridades temáticas) en el debate público en las redes. En este trabajo se aporta un enfoque complementario desde el análisis de la recepción de esos mensajes por parte de la audiencia. En las redes, con respecto a los medios tradicionales, los estudios de recepción cobran si cabe más importancia, pues debido a los flujos de la comunicación on line es más habitual que el receptor interactúe con el resto de públicos en respuesta a un determinado mensaje. Además, las posibilidades de análisis de datos que ofrece Internet y las redes sociales permiten estudios muy precisos sobre el efecto de los mensajes en la audiencia.

De acuerdo a la dinámica de funcionamiento de las redes sociales se sabe que estas premian los perfiles que publican más mensajes. Sin embargo, el nivel de interacción y engagement de una cuenta es mucho más valioso que el número de seguidores que tenga. Los resultados de este estudio también lo muestran. El estudio se ha basado en la actividad en redes sociales (Facebook, Instagram, Twitter y YouTube), en las que se ha medido el nivel de interacción y engagement a lo largo de un mes, monitorizando los posts de los candidatos y partidos que diariamente han logrado una mayor visibilidad en redes. Y es el partido Vox el 
que logra los mejores resultados de sus mensajes en redes sociales.

Del análisis de los resultados se observa que el partido Vox presenta una estrategia de comunicación en redes con mayor efectividad que el resto de partidos, al lograr el mayor nivel tanto de interactividad, como de engagement con la audiencia en todas las redes analizadas. Tan solo en Youtube se observa un mejor resultado del Partido Popular. Asimismo, a diferencia de otros países, se constata que en España las cuentas personales de los candidatos a penas logran aproximarse a la viralidad de las de sus partidos. Otra de las conclusiones señaladas en este artículo que requerirán de futuros estudios para ahondar en las causas es la importancia de las redes sociales de naturaleza más visual, Instagram y Youtube que, aunque cuentan con un menor número de seguidores, generan altos niveles de engagement.

Como afirman Rúas y Casero-Ripollés (2018), "la llegada de las redes sociales ha reconfigurado todo aquello que se daba por establecido". Efectivamente, en este trabajo también se pone de manifiesto que la esfera pública digital funciona con reglas propias, en las que la democratización de los flujos informativos, permite invertir patrones con respecto al modelo tradicional. En las redes sociales no se observa esa menor visibilidad que tienen algunos partidos y formaciones en los medios de comunicación tradicionales, debido a la menor base sociológica a la que representan. Este hecho, requerirá de futuros estudios y análisis porque en la medida en que el uso de redes sociales con finalidad informativa siga creciendo, la importancia de la esfera pública digital seguirá ganando en importancia.

Por último, señalar que, dado que el objetivo es un análisis del resultado de viralidad en redes de los mensajes, se excluyen cuestiones de análisis de contenido de estos mensajes. A pesar de que algunos estudios han concluido que no existen diferencias significativas entre el contenido del tweet y su nivel de engagement (Carrasco-Polaino, Villar-Cirujano; Martín-Cárdaba: 2019:10), sin embargo, se sugiere el interés de cruzar en futuras investigaciones los análisis de resultados de visibilidad como el aquí realizado, con análisis de contenido de mensajes, lo cuál supondría un avance en el estudio de las estrategias de contenido en redes, lo que a su vez sería muy esclarecedor para la comprensión de la comunicación política en la esfera digital.

\section{REFERENCIAS}

ABEJÓN-MENDOZA, PALOMA; MAYORAL-SÁNCHEZ, JAVIER (2017). "Persuasión a través de Facebook de los candidatos en las elecciones generales de 2016 en España”. El profesional de la información, v. 26, n. 5, pp. 928-936. https://doi.org/10.3145/ epi.2017.sep.14

ALONSO, MARÍA (2016). “Opinión pública y web 2.0. Las redes digitalizan el barómetro político en España”. Revista mexicana de opinión pública, v. 21, pp. 95-113. https://doi.org/10.1016/j.rmop.2016.07.004

AMADO, A., \& TARULLO, R. (2015). Las redes sociales en la comunicación política: ¿ comunicación unidireccional o conversacional?. Contratexto, v. 24, pp. 97-111. 
BERROCAL-GONZALO, SALOMÉ; MARTÍN-JIMÉNEZ, VIRGINIA; GILTORRES, ALICIA (2017). "Líderes políticos en YouTube: información y politainment en las elecciones generales de 2016 (26J) en España”. El profesional de la información, v. 26, n. 5, pp. 937-946. https://doi.org/10.3145/epi.2017.sep.15

CAMPOS-DOMÍNGUEZ, EVA; GARCÍA-OROSA, BERTA (2018). “Comunicación algorítmica en los partidos políticos: automatización de producción y circulación de mensajes”. El profesional de la información, v. 27, n. 4, pp. 769-777. https://doi.org/10.3145/ epi.2018.jul.06

CARRASCO-POLAINO, RAFAEL; VILLAR-CIRUJANO, ERNESTO; MARTÍNCÁRDABA, MIGUEL-ÁNGEL (2019). "Redes, tweets y engagement: análisis de las bibliotecas universitarias españolas en Twitter". El profesional de la información, v.28, n. 4, pp. 1699-2407. https://doi.org/10.1080/19322909.2010.487766.

CASERO-RIPOLLÉS, ANDREU (2015). "Estrategias y prácticas comunicativas del activismo político en las redes sociales en España". Historia y comunicación social, v. 20, n. 2, pp .533-548

CONGOSTO, MARÍA (2015). “Elecciones Europeas 2014: Viralidad de los mensajes en Twitter. Redes". Revista hispana para el análisis de redes sociales, v. 26, n. 1, pp. 23-52. https://doi.org/10.5565/rev/redes.529

CRIADO, JUAN IGNACIO; MARTINEZ, GUADALUPE; SILVÁN, AITOR (2012) "Social Media for Political Campaigning. The Use of Twitter by Spanish Mayors in 2011 Local Elections". En: Reddick, Christopher y Aikins, Stephen (Eds.). Web 2.0 Technologies and Democratic Governance. New York: Springer, pp. 219-232. Doi: 10.1007/978-1-46141448-3_1

ENLI, GUNN; SKOGERBØ, ELI (2013). "Personalized campaigns in partycentred politics: Twitter and Facebook as arenas for political communication". Information, communication \& society, v. 16, n. 5, pp. 757-774. DOI: 10.1080/1369118X.2013.782330

GÓMEZ-CALDERÓN, BERNARDO; ROSES, SERGIO; PANIAGUA-ROJANO, FRANCISCO-JAVIER (2017). "La campaña en 140 caracteres. Empleo de Twitter por parte de los candidatos de los partidos mayoritarios ante las elecciones generales de 2016 en España”. El profesional de la información, v. 26, n. 5, pp. 816 823. https://doi.org/10.3145/ epi.2017.sep.04

GULATI, GIRISH; WILLIAMS, CHRISTINE (2010). “Congressional Candidates' Use of YouTube in 2008: Its Frequency and Rationale". Journal of Information Technology \& Politics, v. 7, n. 2, pp. 93-109. https://doi.org/10.1080/19331681003748958

HERNÁNDEZ-SANTAOLALLA, VICTOR; SOLA-MORALES, SALOMÉ (2019). "Postverdad y discurso intimidatorio en Twitter durante el referéndum catalán del 1-O". 
Observatorio (OBS*), v. 13, n. 1. DOI: 10.15847/obsOBS13120191356

JIVKOVA-SEMOVA, DIMITRINA; REQUEIJO-REY, PAULA; PADILLACASTILLO, GRACIELA (2017). "Usos y tendencias de Twitter en la campaña a elecciones generales españolas del 20D de 2015: hashtags que fueron trending topic". El profesional de la información, v. 26, n. 5, pp. 824-837. https://doi.org/10.3145/epi.2017.sep.05

LARSSON, ANDERS; HALLVARD, MOE (2010). “Studying political microblogging: Twitter users in the 2010 Swedish election campaign". New Media and Society, v. 14, n. 5, pp. 729-747. https://doi.org/10.1177/1461444811422894

LÓPEZ-HERMIDA, ALBERTO; FIERRO-ZAMORA, PEDRO (2016). “Campañas políticas y desafección ciudadana: aproximación desde Chile a los efectos de las actividades electorales en el proceso democrático". Palabra Clave, v. 19, n. 2, pp. 365-397. doi: 10.5294/ pacla.2016.19.2.2 DOI: 10.5294/pacla.2016.19.2.2

MAY, ALBERT. (2008). Campaign 2008: It's on YouTube. Nieman Reports, 62.2 Summer: 24

MOYA SÁNCHEZ, MIGUEL; HERRERA DAMAS SUSANA (2016). “Cómo medir el potencial persuasivo en Twitter: propuesta metodológica”. Palabra Clave, v. 19, n. 3, pp. 838-867. doi: 10.5294/pacla.2016.19.3.7

MOYA SÁNCHEZ, MIGUEL; HERRERA DAMAS SUSANA (2015). Hacia una comunicación política avanzada en la Internet 2.0. Observatorio (OBS*), 9(4), pp. 113-139.

QUEVEDO-REDONDO, RAQUEL; PORTALÉS-OLIVA, MARTA (2017). “Imagen y Comunicación Política en Instagram. Celebrificación de los candidatos a la presidencia del Gobierno". El profesional de la información, v. 26, n. 5, pp. 916-927. doi: https://doi. org/10.3145/epi.2017.sep.13.

PÉREZ-CURIEL, CONCHA; GARCÍA-GORDILLO, MAR (2018). "Política de influencia y tendencia fake en Twitter. Efectos postelectorales (21D) en el marco del Procés en Cataluña”. El profesional de la información, v. 27, n. 5, pp. 1030-1040.

RAMOS, MARINA; FERNÁNDEZ-GÓMEZ, JORGE; PINEDA, ANTONIO (2018). "Follow the closing of the campaign on streaming: The use of Twitter by Spanish political parties during the 2014 European elections". New Media and Society, v. 20, n. 1, pp. 122140. doi: $10.1177 / 1461444816660730$.

RUANO, LUIS; LÓPEZ, JUAN; MOSQUERA, JONATHAN (2018). "La política y lo político en Twitter: Análisis del discurso de los candidatos presidenciales de Colombia". RISTI-Revista Ibérica de Sistemas e Tecnologias de Informação, v. 28, pp. 57-71. http:// dx.doi.org/10.17013/risti.28.57-71 
RÚAS ARAÚJO, XOSÉ; CASERO-RIPOLLÉS, ANDREU (2018). “Comunicación política en la época de la redes sociales: lo viejo y lo nuevo, y más allá". adComunica. Revista Científica de Estrategias, Tendencias e Innovación en Comunicación, v. 16., pp. 2124. doi: http://dx.doi.org/10.6035/2174-0992.2018.16.2

SAID-HUNG, ELÍAS; PRATI, RONALDO; CANCINO-BORBÓN, ANDREA (2017). "La orientación ideológica de los mensajes publicados en Twitter durante el 24M en España”. Palabra Clave, n. 20, v. 1, pp. 213-238. doi: 10.5294/pacla.2017.20.1.10

SANTOS, MARCELO; GOMES, JOSE ANTONIO (2017). "Party ideologies in 140 characters: Twitter use by Brazilian Congressmen". Revista de Administração Pública, v. 51, n. 6, pp. 1041-1057. http://dx.doi.org/10.1590/0034-7612155837

SELVA-RUIZ, DAVID; CARO-CASTAÑO, LUCÍA (2017). "Uso de Instagram como medio de comunicación política por parte de los diputados españoles: la estrategia de humanización en la vieja y la nueva política”. El profesional de la información, v. 26, n. 5, pp. 903-915.https://doi.org/10.3145/epi.2017.sep.12

TUMASJAN, ANDRANIK; SPRENGER, TIMM; SANDER, PHILIPP; WELP, ISABELL (2012). "Predicting Election with Twitter: What 140 Characters Reveal about Political Sentimen”. Social Science Computer Review, n. 30, pp. 229-234. https://doi. org/10.1177/0894439310386557

ZITTEL, THOMAS (2009). "Lost in Technology? Political Parties and Online Campaigning in Germany's Mixed Member Electoral System". Journal of Information Technology and Politics, v. 6, n. 3-4, pp. 298-311. https://doi.org/10.1080/19331680903048832

ZUGASTI-AZAGRA, RICARDO; PÉREZ-GONZÁLEZ，JAVIER (2015). “La interacción política en Twitter: el caso de@ppopular y @ahorapodemos durante la campaña para las elecciones europeas de 2014”. Ámbitos. Revista Internacional de Comunicación, v. 28, pp. 1-14. http://institucional.us.es/ambitos/?p=1697 